\title{
Effect of Corticosteroid Therapy on Long-Term Clinical Outcome and Left Ventricular Function in Patients With Cardiac Sarcoidosis
}

\author{
Toshiyuki Nagai, MD, PhD; Nobutaka Nagano, MD; Yasuo Sugano, MD, PhD; \\ Yasuhide Asaumi, MD, PhD; Takeshi Aiba, MD, PhD; Hideaki Kanzaki, MD; \\ Kengo Kusano, MD, PhD; Teruo Noguchi, MD, PhD; Satoshi Yasuda, MD, PhD; \\ Hisao Ogawa, MD, PhD; Toshihisa Anzai, MD, PhD
}

Background: Cardiac involvement is the worst prognostic determinant in patients with sarcoidosis, but the longterm prognostic significance of corticosteroid therapy for cardiac sarcoidosis (CS) remains unclear.

\begin{abstract}
Methods and Results: We examined 83 consecutive patients diagnosed with CS. Patients were divided into 2 groups based on the presence or absence of corticosteroid therapy at diagnosis. Patients with corticosteroid therapy had lower age and higher rate of positive findings in the myocardium on gallium scintigraphy (Ga) at diagnosis than those without. LVEF, biomarkers, and use of cardiovascular medication were similar between the 2 groups. During the follow-up (7.6 \pm 4.4 years), corticosteroid therapy was associated with fewer long-term adverse events (overall, $\mathrm{P}=0.005$; cardiac death, $\mathrm{P}=0.92$; symptomatic arrhythmias, $\mathrm{P}=0.89$; heart failure admission, $\mathrm{P}<0.0001$ ) and a greater $\%$ increase in LVEF than those without $(7.9 \pm 36.3 \%$ vs. $-16.7 \pm 34.8 \%, \mathrm{P}=0.03)$. On Cox proportional hazards modeling, corticosteroid therapy ( $\mathrm{HR}, 0.41 ; 95 \% \mathrm{Cl}: 0.20-0.89)$ was an independent determinant of long-term adverse event-free survival, but age, sex, LVEF, and Ga findings were not.
\end{abstract}

Conclusions: Corticosteroid therapy might have a beneficial effect on long-term clinical outcome in CS patients, particularly by reduction of heart failure admission and retarding the progression of LV systolic dysfunction. (Circ J 2015; 79: 1593-1600)

Key Words: Cardiac sarcoidosis; Corticosteroid; Left ventricular function; Long-term prognosis

$\mathbf{S}$ arcoidosis is a rare, multisystemic granulomatosis characterized by non-caseating granulomas in the involved organs. Although sarcoidosis primarily involves granuloma formation in lung tissue, cardiac involvement, termed cardiac sarcoidosis (CS), is being increasingly recognized as a determinant of poor outcome.

The clinical manifestations of CS are varied, but are frequently complicated by systolic left ventricular (LV) dysfunction and abnormal electrocardiographic findings that include complete heart block and fatal ventricular arrhythmia. ${ }^{1}$ Granulomas characteristic of sarcoidosis are most commonly observed in the myocardium and conduction system, especially in the interventricular septum (IVS) and free wall of the LV. ${ }^{2-4}$ Thus, the treatment of CS is primarily aimed at reducing inflammation and fibrosis associated with granuloma formation.

Corticosteroids are the gold-standard treatment for CS because of their ability to attenuate the inflammatory response, which is related to the progression of granuloma development. ${ }^{5-13}$ Although corticosteroid therapy may prevent adverse LV remodeling, ${ }^{14}$ the majority of studies conducted to date have been limited to single case reports ${ }^{8,10}$ or relatively small population studies $^{14}$ assessing short-term clinical outcome. A previous retrospective study showed that CS patients who received corticosteroid therapy, specifically patients with preserved LV systolic function, had lower mortality compared with untreated patients, ${ }^{15}$ but in that study, all untreated patients were autopsy subjects and the diagnosis of CS had not been made before autopsy. Therefore, it remains unclear whether corticosteroid therapy

Received November 24, 2014; revised manuscript received February 26, 2015; accepted March 11, 2015; released online April 16, 2015 Time for primary review: 22 days

Department of Cardiovascular Medicine, National Cerebral and Cardiovascular Center, Suita (T. Nagai, N.N., Y.S., Y.A., T. Aiba, H.K., K.K., T. Noguchi, S.Y., H.O., T. Anzai); Department of Cardiovascular Medicine, Graduate School of Medical Sciences, Kumamoto University, Kumamoto (H.O.), Japan

The first two authors contributed equally (T. Nagai, N.N.).

Mailing address: Toshihisa Anzai, MD, PhD, Department of Cardiovascular Medicine, National Cerebral and Cardiovascular Center, 5-7-1

Fujishiro-dai, Suita 565-8565, Japan. E-mail: anzai@ ncvc.go.jp

ISSN-1346-9843 doi:10.1253/circj.CJ-14-1275

All rights are reserved to the Japanese Circulation Society. For permissions, please e-mail: cj@j-circ.or.jp 


\begin{tabular}{|c|c|c|c|c|}
\hline Variable & $\begin{array}{l}\text { Overall } \\
(n=83)\end{array}$ & $\begin{array}{l}\text { Corticosteroid } \\
\qquad(n=67)\end{array}$ & $\begin{array}{l}\text { No corticosteroid } \\
\qquad(n=16)\end{array}$ & P-value \\
\hline Age (years) & $60 \pm 12$ & $59 \pm 11$ & $67 \pm 11$ & 0.01 \\
\hline Female & $59(71)$ & $49(73)$ & $10(63)$ & 0.41 \\
\hline \multicolumn{5}{|l|}{ Clinical history } \\
\hline Hypertension & $14(17)$ & $11(16)$ & $3(19)$ & 0.82 \\
\hline Dyslipidemia & $19(23)$ & $15(22)$ & $4(25)$ & 0.82 \\
\hline Diabetes mellitus & $5(6)$ & $4(6)$ & $1(6)$ & 0.97 \\
\hline Smoking & $16(20)$ & $12(18)$ & $4(27)$ & 0.47 \\
\hline Cerebral infarction & $6(7)$ & $4(6)$ & $2(13)$ & 0.40 \\
\hline Advanced AV block & $33(40)$ & $26(39)$ & $7(44)$ & 0.72 \\
\hline VT/VF & $24(29)$ & $20(30)$ & $4(25)$ & 0.70 \\
\hline $\mathrm{CHF}$ & $11(13)$ & $9(13)$ & $2(13)$ & 0.92 \\
\hline \multicolumn{5}{|l|}{ Organ involvement } \\
\hline Lung & $50(60)$ & $38(57)$ & $12(75)$ & 0.17 \\
\hline Skin & $12(14)$ & $10(15)$ & $2(13)$ & 0.80 \\
\hline Eye & $31(37)$ & $22(33)$ & $9(56)$ & 0.09 \\
\hline No. involved organs & $2.1 \pm 0.7$ & $2.1 \pm 0.7$ & $2.4 \pm 0.7$ & 0.08 \\
\hline
\end{tabular}

Data given as mean $\pm \mathrm{SD}$ or $\mathrm{n}(\%)$. AV, atrioventricular; $\mathrm{CHF}$, congestive heart failure; $\mathrm{VF}$, ventricular fibrillation; $\mathrm{VT}$, ventricular tachycardia.

at the point of diagnosis is beneficial.

To clarify the effect of corticosteroid therapy for CS, we evaluated its long-term prognostic significance in a relatively large cohort of CS patients.

\section{Methods}

\section{Subjects}

We examined 127 consecutive patients with clinically diagnosed CS who were admitted between 1990 and 2012. Patients who had coronary artery disease or who did not meet the diagnostic criteria described in the 2006 revised version of the Japanese Ministry of Health and Welfare (JMHW) guidelines for $\mathrm{CS}^{16,17}$ were excluded. Briefly, according to the guidelines, a definite diagnosis of CS was made on the basis of histological findings of endomyocardial biopsy or clinical and/or histological findings meeting clinical cardiac criteria, with extracardiac involvement of at least 1 organ. Finally, 83 patients were included in this study. The study protocol was in agreement with the guidelines of the institutional Ethics Committee (M25-047).

\section{Study Protocol}

We collected the following data: age, sex, coronary risk factors, cardiovascular medication, baseline fatal ventricular arrhythmias, advanced atrioventricular (AV) block, congestive heart failure, catheter ablation for ventricular tachyarrhythmias, cardiac resynchronization therapy (CRT), implantation of permanent pacemaker or implantable cardioverter defibrillator (ICD), extracardiac organ involvement, and imaging findings including gallium (Ga) scintigraphy, ${ }^{18} \mathrm{~F}$-fluorodeoxy glucose-positron emission tomography (FDG-PET), and late gadolinium enhancement-cardiac magnetic resonance (LGE-CMR), at the time of diagnosis. Regarding FDG-PET, specific focal uptake of FDG was defined as positive. The presence of LGE on CMR was defined as any hyper-enhancement in the myocardium. Ga, FDG-PET and LGE-CMR findings were determined by the consensus of 2 experienced radiologists. Venous blood samples were serially obtained to measure plasma angiotensin- converting enzyme (ACE) activity, lysozyme and brain natriuretic peptide (BNP) level, hemoglobin, and serum creatinine and C-reactive protein (CRP) level.

\section{Echocardiography}

Echocardiography was serially performed at the time of diagnosis of CS and during the follow-up period. LV end-diastolic and end-systolic dimensions, thinning of the IVS and LV ejection fraction (LVEF) determined on modified Simpson's method were evaluated. All echocardiographic findings were interpreted by 2 experienced cardiologists.

\section{Study Endpoint and Long-Term Follow-up}

The study endpoint was a composite of all-cause death, heart failure requiring admission, and symptomatic arrhythmia, which was defined as ventricular tachyarrhythmia with clinical symptoms and requiring admission, appropriate ICD treatment for termination of ventricular fibrillation or sustained ventricular tachycardia, or bradyarrhythmias requiring pacemaker implantation. Regarding corticosteroid therapy, initial dose, minimum maintenance dose, and occurrence of major adverse effects (new-onset diabetes mellitus, femoral head necrosis, cataract, osteoporosis and severe sepsis) were also evaluated. Followup data were collected by direct contact at an outpatient clinic or by telephone or mail.

\section{Statistical Analysis}

Continuous data are expressed as mean \pm SD. Continuous variables were compared using unpaired t-test or non-parametric means test. Categorical variables are reported as frequencies with percentages, and were compared between the 2 groups using chi-squared test. Temporal changes in certain variables were statistically analyzed on 1-way analysis of variance for repeated measures. Long-term event-free survival was estimated using Kaplan-Meier curves, and log-rank (Mantel-Cox) test was used to assess differences according to the presence or absence of corticosteroid therapy at the time of diagnosis. Cox proportional hazard model analysis, which included determinant factors with $\mathrm{P} \leq 0.10$ on univariate analysis, was used to 


\begin{tabular}{|lcccc|}
\hline \multicolumn{1}{c}{ Table 2. Baseline Imaging Findings } & Overall & $\begin{array}{c}\text { Corticosteroid } \\
\mathbf{( n = 6 7 )}\end{array}$ & $\begin{array}{c}\text { No corticosteroid } \\
\mathbf{( n = 1 6 )}\end{array}$ & P-value \\
$\begin{array}{l}\text { Echocardiography } \\
\text { LVDd (mm) }\end{array}$ & $56.7 \pm 9.9$ & $56.1 \pm 10.2$ & $58.9 \pm 8.5$ & 0.32 \\
LVDs (mm) & $44.6 \pm 12.7$ & $43.9 \pm 12.7$ & $47.4 \pm 12.6$ & 0.33 \\
LVEF (\%) & $35.8 \pm 14.5$ & $35.9 \pm 14.4$ & $35.2 \pm 15.8$ & 0.86 \\
Basal IVS thinning & $40(49)$ & $33(50)$ & $7(44)$ & 0.65 \\
Other imaging modalities & & & & 0.02 \\
Ga scintigram positive, positive/n & $45 / 79(57)$ & $41 / 65(63)$ & $4 / 14(29)$ & 0.06 \\
FDG-PET positive, positive/n & $42 / 63(67)$ & $38 / 54(70)$ & $4 / 9(44)$ & 0.16 \\
LGE-CMR positive, positive/n & $24 / 27(89)$ & $19 / 21(90)$ & $5 / 6(83)$ & \\
\hline
\end{tabular}

Data given as mean \pm SD or $n(\%)$. FDG-PET, ${ }^{18} \mathrm{~F}$-fluorodeoxy glucose-positron emission tomography; $\mathrm{Ga}$, gallium; IVS, interventricular septum; LGE-CMR, late gadolinium enhancement-cardiac magnetic resonance; LVDd, left ventricular diastolic diameter; LVDs, left ventricular systolic diameter; LVEF, left ventricular ejection fraction.

\begin{tabular}{|c|c|c|c|c|}
\hline Variable & $\begin{array}{l}\text { Overall } \\
(n=83)\end{array}$ & $\begin{array}{l}\text { Corticosteroid } \\
\qquad(n=67)\end{array}$ & $\begin{array}{l}\text { No corticosteroid } \\
\qquad(n=16)\end{array}$ & P-value \\
\hline \multicolumn{5}{|l|}{ Laboratory data } \\
\hline Hemoglobin (g/dl) & $13.2 \pm 1.4$ & $13.3 \pm 1.4$ & $12.7 \pm 1.0$ & 0.13 \\
\hline Serum creatinine (mg/dl) & $0.8 \pm 0.2$ & $0.8 \pm 0.2$ & $0.8 \pm 0.3$ & 0.26 \\
\hline ACE (IU/L) & $15.1 \pm 8.4$ & $15.5 \pm 8.4$ & $13.6 \pm 8.6$ & 0.44 \\
\hline Lysozyme (IU/L) & $10.1 \pm 5.2$ & $10.2 \pm 4.9$ & $9.6 \pm 5.9$ & 0.67 \\
\hline BNP $(p g / m l)$ & $249 \pm 242$ & $255 \pm 247$ & $230 \pm 228$ & 0.74 \\
\hline $\mathrm{CRP}(\mathrm{mg} / \mathrm{dl})$ & $0.19 \pm 0.17$ & $0.19 \pm 0.20$ & $0.20 \pm 0.17$ & 0.86 \\
\hline \multicolumn{5}{|l|}{ Medication } \\
\hline Corticosteroid induction dose (mg/day) & $29.5 \pm 4.0$ & $29.5 \pm 4.0$ & 0 & \\
\hline Other immunosuppressants & $2(2)$ & $2(3)$ & $0(0)$ & 0.48 \\
\hline ACE inhibitors & $25(30)$ & $22(33)$ & $3(19)$ & 0.27 \\
\hline ARB & $19(23)$ & $14(21)$ & $5(31)$ & 0.39 \\
\hline$\beta$-blockers & $40(48)$ & $31(46)$ & $9(56)$ & 0.47 \\
\hline Diuretics & $33(40)$ & $26(39)$ & $7(44)$ & 0.72 \\
\hline Statins & $20(24)$ & $13(19)$ & $7(44)$ & 0.05 \\
\hline Amiodarone & $13(16)$ & $9(13)$ & $4(25)$ & 0.25 \\
\hline Other anti-arrhythmic agents & $21(25)$ & $16(24)$ & $5(31)$ & 0.55 \\
\hline VT-ABL & $12(14)$ & $8(12)$ & $4(25)$ & 0.18 \\
\hline CRT & $10(12)$ & $6(9)$ & $4(25)$ & 0.08 \\
\hline Pacer/ICD & $49(59)$ & $37(55)$ & $12(75)$ & 0.14 \\
\hline
\end{tabular}

Data given as mean $\pm \mathrm{SD}$ or $\mathrm{n}(\%)$. ACE, angiotensin-converting enzyme; ARB, angiotensin receptor blocker; BNP, brain natriuretic peptide; CRP, C-reactive protein; CRT, cardiac resynchronization therapy; ICD, implantable cardioverter defibrillator; VT-ABL, ventricular tachycardia ablation.

assess the association of these factors with adverse events. All statistical analysis was performed using SPSS 13.0 for Windows (SPSS, Chicago, IL, USA). Statistical significance was defined as $\mathrm{P}<0.05$.

\section{Results}

\section{Patient Characteristics}

Corticosteroid therapy was given in 67 CS patients (81\%). Patients with corticosteroid therapy had lower age at the time of diagnosis than those without. In untreated patients, the reasons for withholding corticosteroid therapy were negative Ga scintigraphy and/or FDG-PET, suggesting the absence of active inflammation in the myocardium $(n=7)$, patient refusal $(n=5)$, active infectious disease $(n=2)$, negative endomyocardial biopsy $(\mathrm{n}=1)$, and unknown reason $(\mathrm{n}=1)$.
No significant difference was detected between the 2 groups with respect to sex, incidence of advanced AV block, ventricular tachyarrhythmia, or congestive heart failure at the time of diagnosis. The prevalence of traditional cardiovascular risk factors and extracardiac organ involvement of sarcoidosis was similar in the 2 groups (Table $\mathbf{1}$ ).

\section{Imaging Findings}

Baseline LV end-diastolic dimension, end-systolic dimension, LVEF, and prevalence of basal IVS thinning did not significantly differ between the 2 groups (Table 2). Although positive Ga scintigram was more common in patients with corticosteroid therapy than in those without, the rates of positive PDG-PET and LGE-CMR were similar between the 2 groups. 
A

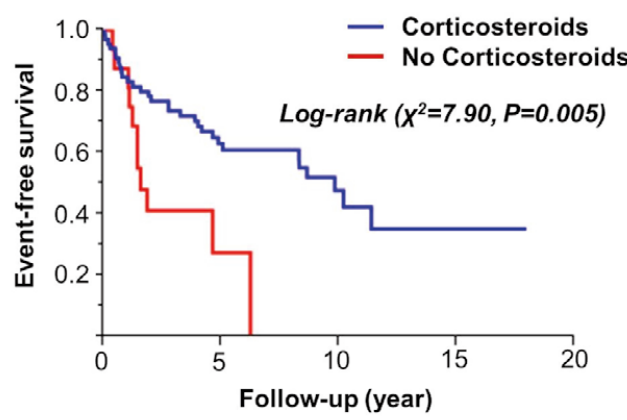

C

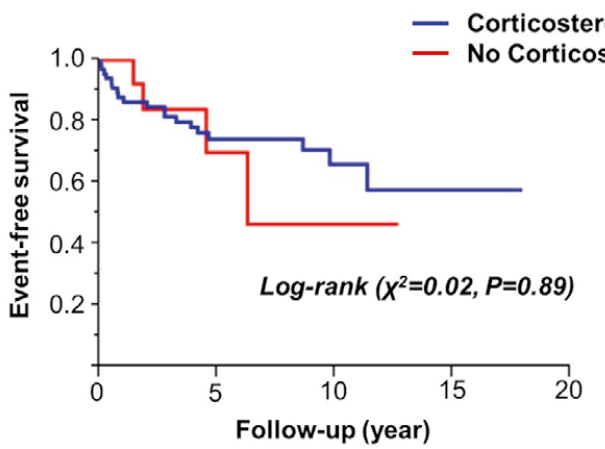

B

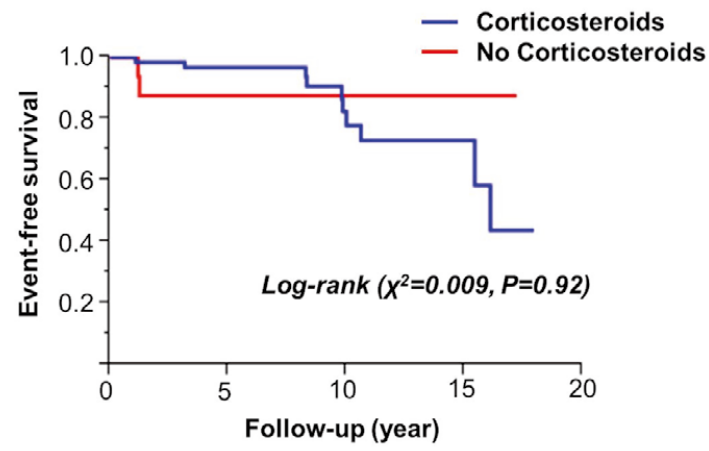

D

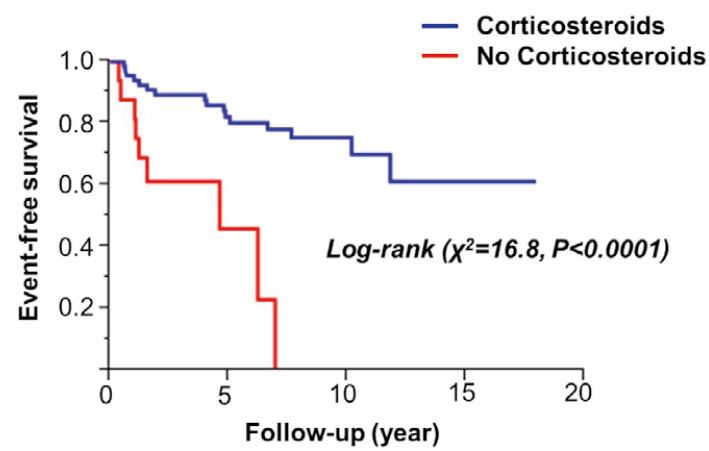

Figure 1. Kaplan-Meier analysis of long-term clinical outcome according to presence of corticosteroid therapy. (A) Composite of all-cause death, symptomatic arrhythmias, and heart failure requiring admission; (B) cardiac death; (C) symptomatic arrhythmias; (D) heart failure requiring admission.

\section{Laboratory Data and Medication}

Laboratory data, including plasma ACE activity, lysozyme and $\mathrm{BNP}$, hemoglobin, and serum creatinine and CRP at the time of diagnosis, were similar between patients with and without corticosteroid therapy.

Use of cardiovascular medication, including $\beta$-blockers, ACE inhibitors or angiotensin receptor blockers, diuretics, statins, amiodarone, and other anti-arrhythmic agents, catheter ablation for ventricular tachyarrhythmias, CRT, and implantation of a permanent pacemaker or ICD were similar between the 2 groups (Table 3).

In patients who received corticosteroid therapy, the initial dose was $29.5 \pm 4.0 \mathrm{mg}$ prednisolone, and few patients received other immunosuppressants (2\%). The use of other immunosuppressants was similar between the 2 groups (Table 3 ).

\section{Long-Term Clinical Outcome}

Patients were followed for $7.6 \pm 4.4$ years. On Kaplan-Meier analysis, corticosteroid therapy started at the time of diagnosis was associated with a lower incidence of composite adverse events (all-cause death, symptomatic arrhythmias, and heart failure requiring admission) during the observation period (Figure 1A). Although corticosteroid therapy was not a significant determinant of cardiac death or symptomatic arrhythmias (Figures 1B,C), it was a negative determinant of heart failure requiring admission (Figure 1D).

On univariate Cox proportional hazards model analysis for determinants of composite adverse events, absence of corticosteroid therapy at the time of diagnosis and higher age were the only variables with $\mathrm{P} \leq 0.10$. The other examined variables, including sex, extracardiac involvement, $\mathrm{LVEF}<35 \%$ (median), positive $\mathrm{Ga}$ and/or FDG-PET findings, hemoglobin $<13 \mathrm{~g} / \mathrm{dl}$, serum creatinine $\geq 0.8 \mathrm{mg} / \mathrm{dl}$ (median), ACE $\geq 14 \mathrm{IU} / \mathrm{L}$ (median), lysozyme $\geq 9 \mu \mathrm{g} / \mathrm{L}$ (median), and BNP $\geq 150 \mathrm{pg} / \mathrm{ml}$ (median), were not significant. In the multivariate Cox proportional hazards analysis model, corticosteroid therapy at the time of diagnosis was confirmed to be an independent determinant of composite adverse events (Table 4).

\section{Change of LV Systolic Function on Long-Term Corticosteroid Therapy}

Patients with corticosteroid therapy had a significantly greater $\%$ increase in LVEF from baseline to follow-up $(6.8 \pm 4.1$ and $4.1 \pm 3.4$ years, respectively) than those without $(7.9 \pm 36.3 \%$ vs. $-16.7 \pm 34.8 \%, P=0.03$; Figure 2A). These changes tended to be different between patients with and without corticosteroid therapy for those with higher LVEF ( $\geq 35 \%)$, but not for those with lower LVEF $(<35 \%$; Figures $2 B, C)$. 


\begin{tabular}{|c|c|c|c|c|c|c|}
\hline \multirow{2}{*}{ Variable } & \multicolumn{3}{|c|}{ Univariate } & \multicolumn{3}{|c|}{ Multivariate } \\
\hline & HR & $95 \% \mathrm{Cl}$ & P-value & HR & $95 \% \mathrm{Cl}$ & P-value \\
\hline Corticosteroid therapy at diagnosis & 0.37 & $0.18-0.79$ & 0.012 & 0.41 & $0.20-0.89$ & 0.026 \\
\hline Age $\geq 63$ years & 1.67 & $0.88-3.27$ & 0.09 & 1.42 & $0.72-2.85$ & 0.31 \\
\hline Female & 1.28 & $0.66-2.68$ & 0.47 & - & - & - \\
\hline Organ involvement $\geq 2$ organs & 1.35 & $0.57-3.97$ & 0.52 & - & - & - \\
\hline LVEF $<35 \%$ & 1.56 & $0.80-3.05$ & 0.19 & - & - & - \\
\hline Ga scintigram positive & 0.94 & $0.50-1.79$ & 0.85 & - & - & - \\
\hline FDG-PET positive & 0.90 & $0.44-1.97$ & 0.79 & - & - & - \\
\hline Hemoglobin $<13 \mathrm{~g} / \mathrm{dl}$ & 1.48 & $0.79-2.76$ & 0.22 & - & - & - \\
\hline Serum creatinine $\geq 0.8 \mathrm{mg} / \mathrm{dl}$ & 1.05 & $0.51-2.03$ & 0.88 & - & - & - \\
\hline$A C E \geq 14 \mathrm{IU} / \mathrm{L}$ & 0.91 & $0.48-1.70$ & 0.77 & - & - & - \\
\hline Lysozyme $\geq 9 \mu \mathrm{g} / \mathrm{L}$ & 1.41 & $0.75-2.73$ & 0.29 & - & - & - \\
\hline $\mathrm{BNP} \geq 150 \mathrm{pg} / \mathrm{ml}$ & 1.51 & $0.73-3.18$ & 0.26 & - & - & - \\
\hline CRT & 1.87 & $0.92-3.59$ & 0.18 & & & \\
\hline
\end{tabular}

$\mathrm{Cl}$, confidence interval; HR, hazard ratio. Other abbreviations as in Tables 2,3.
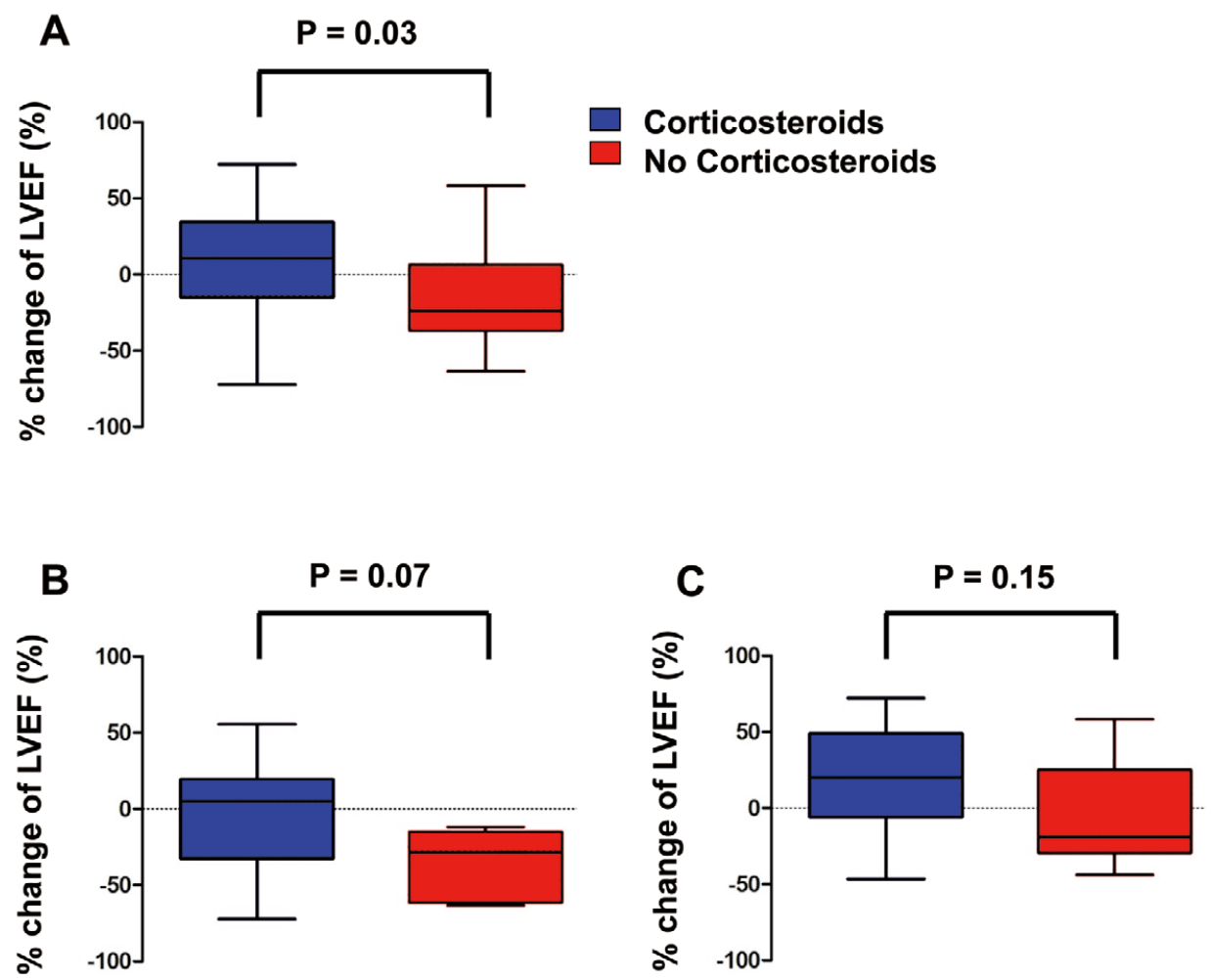

Figure 2. Change of left ventricular ejection fraction (LVEF) during long-term follow-up. (A) Overall; (B) LVEF $\geq 35 \%$ (median) at baseline; (C) LVEF $<35 \%$ at baseline.

\section{Adverse Effects of Corticosteroid Therapy}

The mean treatment duration was $7.1 \pm 4.6$ years and the mean minimum maintenance dose of corticosteroid therapy was $7.3 \pm 4.3 \mathrm{mg} /$ day. In the corticosteroid therapy group, $28 \%$ of patients had major adverse effects, including new-onset diabetes mellitus and osteoporosis (15\% and $18 \%$, respectively, Table 5), but no patient died from the adverse effects of corticosteroid therapy.

\section{Discussion}

In the present cohort study of CS patients, corticosteroid therapy had long-term beneficial effects, particularly in prevention of heart failure admission and preservation of LV systolic function. Notably, the presence of positive findings on Ga scintigraphy and/or FDG-PET did not determine long-term clinical outcome. This suggests that initiating corticosteroid therapy at 


$\begin{aligned} & \text { Table 5. Major Adverse Effects of Long-Term Corticosteroid } \\ & \text { Therapy }\end{aligned}$
\[ \begin{array}{c}\text { Variable } \\ \text { Corticosteroid } \\ (\mathbf{n}=67)\end{array} \]
Corticosteroid therapy
Duration (years)
Minimum maintenance dose (mg/day)
Adverse effects
Overall
New-onset diabetes mellitus
Femoral head osteonecrosis
Cataract
Osteoporosis
Sepsis

Data given as mean $\pm \mathrm{SD}$ or $\mathrm{n}(\%)$.

the time of CS diagnosis may improve the clinical outcome, even in patients without evidence of active myocardial inflammation on diagnostic imaging.

\section{Effect of Corticosteroid Therapy on Long-Term Clinical Outcome}

The present findings are consistent with previous studies that showed that corticosteroid therapy improved CS symptoms related to $\mathrm{LV}$ dysfunction and fatal arrhythmias. ${ }^{5,8-12,15,18}$ Yazaki et al showed that CS patients who were treated with corticosteroids had lower mortality compared with untreated patients in whom CS was diagnosed at autopsy. ${ }^{15}$ ChapelonAbric et al also reported that $87 \%$ of CS patients who received corticosteroid therapy had clinical improvement, although the study population was relatively small. ${ }^{5}$ Furthermore, several case reports have documented the effectiveness of corticosteroid therapy for improving LV function, atrial arrhythmias, advanced AV block, and electrocardiographic abnormalities in CS patients, even in those with severe myocardial damage. ${ }^{8-12,18}$ The present study examined clinical outcomes of CS patients for a longer follow-up period and grouped patients into treated and untreated groups at the diagnosis of CS, rather than identifying untreated patients on post-mortem diagnosis. Based on the present findings, corticosteroid therapy may reduce the incidence of heart failure admission and preserve long-term LV systolic function in patients with CS.

Regardless of the significant reduction of heart failure admission in patients with corticosteroid therapy, the incidence of cardiac death and symptomatic arrhythmias did not markedly differ from that in untreated patients. ICD device implantation and catheter ablation for sustained ventricular tachycardia at the time of diagnosis, however, tended to be more common in untreated patients compared with treated patients. These aggressive treatments may obscure the effect of corticosteroid therapy in preventing sudden cardiac death and the development of symptomatic arrhythmias.

\section{Corticosteroid Therapy and LV Systolic Function}

Previous case reports noted an approximately $20 \%$ improvement in LVEF after 4 weeks of prednisolone treatment. ${ }^{8,10}$ Chiu et al reported that corticosteroid therapy in CS patients with baseline LVEF $\geq 55 \%$ had preventive effects on LV remodeling and systolic dysfunction, although few benefits of therapy on LV function were detected in those with baseline LVEF $<30 \% .{ }^{14}$ In that study, however, the serial change of LV function in the untreated CS group was not examined. In the pres- ent study, CS patients who received corticosteroid therapy had greater improvement of LV systolic function compared with those without, especially in patients with baseline LVEF $\geq 35 \%$. These findings suggest that severe LV dysfunction in CS may represent end-stage myocardial fibrosis, which is refractory to corticosteroid therapy. Corticosteroid therapy may need to be started in the earlier stages of CS to ensure a beneficial effect on LV systolic function.

\section{Myocardial Inflammation and Corticosteroid Therapy}

In CS patients, active inflammation often involves the basal region of the septum and the $\mathrm{LV}$ wall ${ }^{19}$ and is thought to be associated with the development of sudden cardiac death., ${ }^{1,4,20}$ Corticosteroid therapy has been shown to markedly reduce the size and number of regions of inflammation, ${ }^{21-23}$ and myocardial lesions without active inflammation appear to respond poorly to corticosteroids. ${ }^{24}$ Thus, it has been proposed that the mechanisms underlying the effects of corticosteroids appear to be related to the attenuation of inflammation associated with granuloma formation in myocardial tissue. ${ }^{14}$ Corticosteroids are reported to suppress the expression of many inflammatory cytokines including interleukin-1, 6, 19, interferon- $\gamma$ and tumor necrosis factor- $\alpha$, which play important roles in the development of sarcoid granuloma, ${ }^{25,26}$ and also to downregulate T-cell immunity, diminish T-cell activation, and enhance apoptosis of activated T-cells in an experimental pulmonary inflammation model. ${ }^{27} \mathrm{~A}$ number of case reports and small population studies have indicated that myocardial Ga uptake reflects the activity of sarcoid granulomas ${ }^{28,29}$ and may be a useful indicator of the response to corticosteroid treatment. ${ }^{24,30}$ One case report, however, showed that LV function was not significantly improved by corticosteroid therapy despite attenuation of myocardial inflammation on PET. ${ }^{31}$ These discrepant results may be due to the limited accuracy of the current imaging modalities. The sensitivity of Ga scintigraphy in patients with $\mathrm{CS}$ is relatively low and was reported to range from $18 \%$ to $50 \% .{ }^{32-35}$ Although the sensitivity of FDG-PET is superior to that of other modalities (82-100\%), its specificity was reported to be insufficient for definite diagnosis, ${ }^{32-40}$ and the present study indicated that active inflammation on Ga scintigraphy or PET might not be useful for predicting long-term clinical outcome. Therefore, the clinical significance of Ga scintigraphy and PET as surrogate markers of the response to corticosteroid therapy in CS patients is still controversial. Moreover, as mentioned here, the sensitivity and specificity of the 2 imaging modalities are different, and fasting periods and dietary modification were not standardized, thus, the relationship between the active inflammation detected using these modalities and the response to corticosteroid therapy could not be determined. Prospective studies will be needed to determine the clinical usefulness of Ga scintigraphy and PET in CS patients.

\section{Dose and Adverse Effects of Corticosteroids}

Although corticosteroids remain the mainstay of treatment for CS, the optimal initial and maintenance doses remain unclear. The present cohort of patients received an average initial dose of $29.5 \pm 3.8 \mathrm{mg} / \mathrm{day}$, which is lower than the $60-80-\mathrm{mg}$ daily dose of prednisone that has been reported in several case reports. ${ }^{14,15}$ A previous study, however, showed that the clinical outcomes did not markedly differ between patients given either low $(\leq 30-\mathrm{mg})$ or high $\left(\geq 40\right.$-mg) starting doses. ${ }^{15}$ Moreover, a long-term follow-up study showed that 6 of $7 \mathrm{CS}$ patients treated with corticosteroids had adverse effects. ${ }^{12}$ Based on these findings, lower dose corticosteroid therapy is advisable 
for patients with $\mathrm{CS}$ in clinical practice. With regard to the maintenance dose, $7.2 \pm 4.2 \mathrm{mg} /$ day was used in the present study, and a maintenance dose of $10-15 \mathrm{mg}$ was administered every other day after the first 6 months of therapy.

Commonly observed adverse effects of corticosteroid therapy include osteoporosis, hypertension, cataracts, femoral head osteonecrosis, hyperglycemia, and severe infection. ${ }^{6}$ In the present cohort of CS patients, the major adverse effects were new-onset diabetes mellitus and osteoporosis, and there were no deaths attributed to treatment. Thus, corticosteroid therapy appears to be relatively well tolerated in patients with $\mathrm{CS}$, and the selection of initial and maintenance doses must reflect the balance between obtaining a therapeutic response and avoiding induction of the above adverse effects. ${ }^{41}$

\section{Study Limitations}

First, although the study population was relatively large compared with those of previous studies examining the effect of corticosteroid therapy for CS, the present sample size was still small, especially in patients without corticosteroid therapy, thereby limiting the ability to generalize the findings and the statistical power for detecting differences in negative data. Therefore, further prospective studies with a larger population are warranted to make a definitive conclusion with regard to the survival benefit of corticosteroids. Second, because patients without a pacemaker, ICD, or CRT were not always monitored with 24-h electrocardiography, it is possible that the incidence of several tachy- and bradyarrhythmias might have been underestimated. For this reason, one of the study endpoints regarding arrhythmic events was defined as symptomatic ventricular arrhythmias requiring admission, appropriate ICD treatment for termination of ventricular fibrillation or sustained ventricular tachycardia, or bradyarrhythmias requiring permanent pacemaker implantation. Finally, due to the retrospective nature of this study, there was a potential for sampling bias and incomplete data, and further prospective studies in larger populations are warranted.

\section{Conclusions}

Initiating corticosteroid therapy at the time of diagnosis might improve long-term clinical outcome through reduction of heart failure admission and preservation of LV systolic function.

\section{Acknowledgments}

This work was supported by a Grant from the Japan Cardiovascular Research Foundation (T. Anzai), and a Grant-in-Aid for Young Scientists (T. Nagai, 25860630) from the Japan Society for the Promotion of Science.

\section{Disclosures}

No financial supports exist in this study.

\section{References}

1. Roberts WC, McAllister HA Jr, Ferrans VJ. Sarcoidosis of the heart: A clinicopathologic study of 35 necropsy patients (group 1) and review of 78 previously described necropsy patients (group 11). Am J Med 1977; 63: 86-108.

2. Fleming HA. Sarcoid heart disease. Br Heart J 1974; 36: 54-68.

3. Lorell B, Alderman EL, Mason JW. Cardiac sarcoidosis: Diagnosis with endomyocardial biopsy and treatment with corticosteroids. Am J Cardiol 1978; 42: 143-146.

4. Silverman KJ, Hutchins GM, Bulkley BH. Cardiac sarcoid: A clinicopathologic study of 84 unselected patients with systemic sarcoidosis. Circulation 1978; 58: 1204-1211.

5. Chapelon-Abric C, de Zuttere D, Duhaut P, Veyssier P, Wechsler B, Huong DL, et al. Cardiac sarcoidosis: A retrospective study of 41 cases. Medicine (Baltimore) 2004; 83: 315-334.

6. Bussinguer M, Danielian A, Sharma OP. Cardiac sarcoidosis: Diagnosis and management. Curr Treat Options Cardiovasc Med 2012; 14: $652-664$.

7. Umetani K, Ishihara T, Yamamoto K, Sawanobori T, Kohno I, Ijiri $\mathrm{H}$, et al. Successfully treated complete atrioventricular block with corticosteroid in a patient with cardiac sarcoidosis: Usefulness of gallium-67 and thallium-201 scintigraphy. Intern Med 2000; 39: 245 248.

8. Ishikawa T, Kondoh H, Nakagawa S, Koiwaya Y, Tanaka K. Steroid therapy in cardiac sarcoidosis: Increased left ventricular contractility concomitant with electrocardiographic improvement after prednisolone. Chest 1984; 85: 445-447.

9. Fujita N, Hiroe M, Suzuki Y, Sato H, Inoue Y, Sekiguchi M, et al. A case with cardiac sarcoidosis: Significance of the effect of steroids on the reversion of advanced atrioventricular block and myocardial scintigraphic abnormalities. Heart Vessels Suppl 1990; 5: 16-18.

10. Radulescu B, Imperiale A, Germain P, Ohlmann P. Severe ventricular arrhythmias in a patient with cardiac sarcoidosis: Insights from MRI and PET imaging and importance of early corticosteroid therapy. Eur Heart J 2009; 31: 400.

11. Namboodiri N, Stiles MK, Young GD, Sanders P. Electrophysiological features of atrial flutter in cardiac sarcoidosis: A report of two cases. Indian Pacing Electrophysiol J 2012; 12: 284-289.

12. Kato Y, Morimoto S, Uemura A, Hiramitsu S, Ito T, Hishida H. Efficacy of corticosteroids in sarcoidosis presenting with atrioventricular block. Sarcoidosis Vasc Diffuse Lung Dis 2003; 20: 133-137.

13. Hiramitsu S, Morimoto S, Uemura A, Kato Y, Kimura K, Ohtsuki $\mathrm{M}$, et al. National survey on status of steroid therapy for cardiac sarcoidosis in Japan. Sarcoidosis Vasc Diffuse Lung Dis 2005; 22: 210213.

14. Chiu CZ, Nakatani S, Zhang G, Tachibana T, Ohmori F, Yamagishi $\mathrm{M}$, et al. Prevention of left ventricular remodeling by long-term corticosteroid therapy in patients with cardiac sarcoidosis. Am J Cardiol 2005; 95: 143-146.

15. Yazaki Y, Isobe M, Hiroe M, Morimoto S, Hiramitsu S, Nakano T, et al. Prognostic determinants of long-term survival in Japanese patients with cardiac sarcoidosis treated with prednisone. Am J Cardiol 2001; 88: $1006-1010$

16. Hiraga H, Iwai K, Hiroe M, Takada K, Omori F, Yazaki Y, et al. Guideline for the diagnosis of cardiac sarcoidosis: Study report on diffuse pulmonary disease. Japan Ministry Health Welfare 1993: 23-24 (in Japanese).

17. Tsuda T, Ishihara M, Okamoto H, Ohara K, Oritsu M, Sugiura K, et al. Diagnostic criteria and guideline for sarcoidosis - 2006. Jpn J Sarcoidosis Other Granulom Disord 2006; 27: 89-102 (in Japanese).

18. Valantine HA, Tazelaar HD, Macoviak J, Mullin AV, Hunt SA, Fowler $\mathrm{MB}$, et al. Cardiac sarcoidosis: Response to steroids and transplantation. J Heart Transplant 1987; 6: 244-250.

19. Vignaux O. Cardiac sarcoidosis: Spectrum of MRI features. AJR Am J Roentgenol 2005; 184: 249-254.

20. Sekiguchi M, Yazaki Y, Isobe M, Hiroe M. Cardiac sarcoidosis: Diagnostic, prognostic, and therapeutic considerations. Cardiovasc Drugs Ther 1996; 10: 495-510.

21. Vignaux O, Dhote R, Duboc D, Blanche P, Devaux JY, Weber S, et al. Detection of myocardial involvement in patients with sarcoidosis applying T2-weighted, contrast-enhanced, and cine magnetic resonance imaging: Initial results of a prospective study. J Comput Assist Tomogr 2002; 26: $762-767$

22. Vignaux O, Dhote R, Duboc D, Blanche P, Dusser D, Weber S, et al. Clinical significance of myocardial magnetic resonance abnormalities in patients with sarcoidosis: A 1-year follow-up study. Chest 2002; 122: $1895-1901$.

23. Nemeth MA, Muthupillai R, Wilson JM, Awasthi M, Flamm SD. Cardiac sarcoidosis detected by delayed-hyperenhancement magnetic resonance imaging. Tex Heart Inst J 2004; 31: 99-102.

24. Okayama K, Kurata C, Tawarahara K, Wakabayashi Y, Chida K, Sato A. Diagnostic and prognostic value of myocardial scintigraphy with thallium-201 and gallium-67 in cardiac sarcoidosis. Chest 1995; 107: $330-334$

25. Iannuzzi MC, Rybicki BA, Teirstein AS. Sarcoidosis. N Engl J Med 2007; 357: 2153-2165.

26. Grutters JC, van den Bosch JM. Corticosteroid treatment in sarcoidosis. Eur Respir J 2006; 28: 627-636.

27. Khalil N, Whitman C, Zuo L, Danielpour D, Greenberg A. Regulation of alveolar macrophage transforming growth factor-beta secretion by corticosteroids in bleomycin-induced pulmonary inflammation in the rat. J Clin Invest 1993; 92: 1812-1818.

28. Alavi A, Palevsky HI. Gallium-67-citrate scanning in the assessment of disease activity in sarcoidosis. $\mathrm{J} \mathrm{Nucl} \mathrm{Med} \mathrm{1992;} \mathrm{33:} \mathrm{751-755.}$ 
29. Futamatsu H, Suzuki J, Adachi S, Okada H, Otomo K, Ohara T, et al. Utility of gallium-67 scintigraphy for evaluation of cardiac sarcoidosis with ventricular tachycardia. Int J Cardiovasc Imaging 2006; 22: $443-448$.

30. O'Connell JB, Henkin RE, Robinson JA, Subramanian R, Scanlon PJ, Gunnar RM. Gallium-67 imaging in patients with dilated cardiomyopathy and biopsy-proven myocarditis. Circulation 1984; 70: $58-62$.

31. Tadamura E, Yamamuro M, Kubo S, Kanao S, Hosokawa R, Kimura $\mathrm{T}$, et al. Images in cardiovascular medicine: Multimodality imaging of cardiac sarcoidosis before and after steroid therapy. Circulation 2006; 113: e771-e773, doi:10.1161/CIRCULATIONAHA.105.594200.

32. Tadamura E, Yamamuro M, Kubo S, Kanao S, Saga T, Harada M, et al. Effectiveness of delayed enhanced MRI for identification of cardiac sarcoidosis: Comparison with radionuclide imaging. AJR Am J Roentgenol 2005; 185: 110-115.

33. Fields CL, Ossorio MA, Roy TM, Denny DM, Varga DW. Thallium201 scintigraphy in the diagnosis and management of myocardial sarcoidosis. South Med J 1990; 83: 339-342.

34. Kaminaga T, Takeshita T, Yamauchi T, Kawamura H, Yasuda M. The role of iodine-123-labeled 15-(p-iodophenyl)-3R,S-methylpentadecanoic acid scintigraphy in the detection of local myocardial involvement of sarcoidosis. Int J Cardiol 2004; 94: 99-103.
35. Ohira H, Tsujino I, Ishimaru S, Oyama N, Takei T, Tsukamoto E, et al. Myocardial imaging with ${ }^{18} \mathrm{~F}$-fluoro-2-deoxyglucose positron emission tomography and magnetic resonance imaging in sarcoidosis. Eur J Nucl Med Mol Imaging 2008; 35: 933-941.

36. Okumura $\mathrm{W}$, Iwasaki $\mathrm{T}$, Toyama $\mathrm{T}$, Iso $\mathrm{T}$, Arai M, Oriuchi $\mathrm{N}$, et al. Usefulness of fasting ${ }^{18}$ F-FDG PET in identification of cardiac sarcoidosis. J Nucl Med 2004; 45: 1989-1998.

37. Ishimaru S, Tsujino I, Takei T, Tsukamoto E, Sakaue S, Kamigaki $\mathrm{M}$, et al. Focal uptake on ${ }^{18} \mathrm{~F}$-fluoro-2-deoxyglucose positron emission tomography images indicates cardiac involvement of sarcoidosis. Eur Heart J 2005; 26: 1538-1543.

38. Langah R, Spicer K, Gebregziabher M, Gordon L. Effectiveness of prolonged fasting ${ }^{18} \mathrm{~F}$-FDG PET-CT in the detection of cardiac sarcoidosis. J Nucl Cardiol 2009; 16: 801-810.

39. Miyagawa M, Yokoyama R, Nishiyama Y, Ogimoto A, Higaki J, Mochizuki T. Positron emission tomography-computed tomography for imaging of inflammatory cardiovascular diseases. Circ J 2014; 78: $1302-1310$.

40. Ohira H, McArdle B, Cocker MS, deKemp RA, Dasilva JN, Beanlands RS. Current and future clinical applications of cardiac positron emission tomography. Circ J 2013; 77: 836-848.

41. du Bois RM. Corticosteroids in sarcoidosis: Friend or foe? Eur Respir J 1994; 7: 1203-1209. 